\title{
Study of $N^{*}(1440)$ from $J / \Psi$ Decays
}

\author{
B.S.Zou ${ }^{a-d}$, G.X.Peng ${ }^{a, b}$, R.G.Ping ${ }^{b}$, H.C.Chiang ${ }^{a-d}$, W.X.Ma ${ }^{a, b}$ and \\ P.N.Shen ${ }^{a-d}$ \\ a) CCAST(World Lab.), P.O.Box 8730, Beijing 100080; \\ b) Institute of High Energy Physics, Chinese Academy of Science, \\ P.O.Box 918(4), Beijing 100039ף; \\ c) Institute of Theoretical Physics, Chinese Academy of Sciences \\ d) Center of Theoretical Nuclear Physics, National Laboratory of Heavy Ion Accelerator, \\ Lanzhou 730000, P.R.China
}

\begin{abstract}
For $J / \Psi \rightarrow \bar{p} p \pi^{0}$ and $\bar{p} p \pi^{+} \pi^{-}$, the $\pi^{0} p$ and $p \pi^{+} \pi^{-}$systems are limited to be pure isospin $1 / 2$ due to isospin conservation. This is a big advantage in studying $N^{*}$ resonances from $J / \Psi$ decays, compared with $\pi N$ and $\gamma N$ experiments. The process $J / \Psi \rightarrow \bar{p} N^{*}$ or $p \bar{N}^{*}$ provides a new way to probe the internal structure of the $N^{*}$ resonances. Here we report a quark model calculation for $J / \Psi \rightarrow \bar{p} p, \bar{p} N^{*}(1440)$ and $\bar{N}^{*} N^{*}$. The implication for the internal structure of $N^{*}(1440)$ is discussed.
\end{abstract}

\section{PACS: 14.20.Gk; 13.25.Gv; 13.65.+i}

\section{Introduction}

An important source of information about the nucleon internal structure is the properties of nucleon excitation states $N^{*}$ 's, such as their mass spectrum, various production and decay rates [1]. Our present knowledge of this aspect came almost entirely from partialwave analyses of $\pi N$ total, elastic, and charge-exchange scattering data of more than twenty years ago[2]. Since the late 1970's, very little has happened in experimental $N^{*}$ baryon spectroscopy. Considering its importance for the understanding of the baryon structure and for distinguishing various pictures [3] of the nonperturbative regime of QCD, a new generation of experiments on $N^{*}$ physics with electromagnetic probes has recently been started at new facilities such as CEBAF at JLAB, ELSA at Bonn, GRAAL at Grenoble.

A long-standing problem in $N^{*}$ physics is about the nature of the Roper resonance $N^{*}(1440)$. In simple three-quark picture of baryons, it should be the first radial excitation state of the nucleon. But various quark models [3] met difficulties to explain its mass and electromagnetic couplings. It has therefore been suggested [4 to be a gluonic excitation

\footnotetext{
${ }^{1}$ Mailing address
} 
state of the nucleon, i.e., a "hybrid baryon". To establish the gluonic degree of freedom in hadrons is a fascinating challenge in nowadays non-perturbative QCD physics.

Although the existence of the $N^{*}(1440)$ is well-established, its properties, such as mass, width and decay branching ratios etc., still suffer large experimental uncertainties]2. A big problem in extracting information on the $N^{*}(1440)$ from $\pi N$ and $\gamma N$ experiments is the isospin decomposition of $1 / 2$ and $3 / 2$ [5]. As pointed out by one of us[6], the decays of $J / \Psi \rightarrow \bar{p} p \pi^{0}$ and $J / \Psi \rightarrow \bar{p} p \pi^{+} \pi^{-}$provide an ideal place for studying the properties of $N^{*}$ resonances, since in these processes the $\pi^{0} p$ and $p \pi^{+} \pi^{-}$systems are limited to be pure isospin $1 / 2$ due to isospin conservation. Preliminary results from the BES Collaboration on $J / \Psi \rightarrow \bar{p} p \pi^{0}$ show a clear peak structure around $1490 \mathrm{MeV}$ in its $\pi^{0} p$ invariant mass spectrum [7].

The process $J / \Psi \rightarrow \bar{p} N^{*}$ or $p \bar{N}^{*}$ also provides a new way to probe the internal quarkgluon structure of the $N^{*}$ resonances. In the simple three-quark picture of baryons, the process can be described by Fig.1 [8]. In this picture, three quark-antiquark pairs are created independently via a symmetric three-gluon intermediate state with no extra interaction other than the recombination process in the final state to form baryons. This is quite different from the mechanism underlying the $N^{*}$ production from the $\gamma p$ process where the photon couples to only one quark and unsymmetric configuration of quarks is favored. Therefore the processes $J / \Psi \rightarrow \bar{p} N^{*}$ and $\gamma p \rightarrow N^{*}$ should probe different aspects of the quark distributions inside baryons. Since the $J / \Psi$ decay is a glue-rich process, a hybrid $N^{*}$ is expected to have larger production rate than a pure three-quark $N^{*}[9]$.

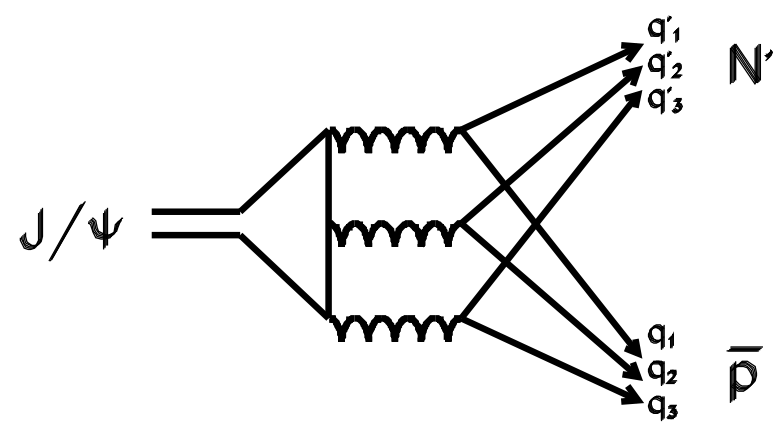

Figure 1: Lowest-order diagram for $J / \Psi \rightarrow \bar{p} N^{\prime}$ with $N^{\prime}$ to be $p$ or $N^{*}$

If $N^{*}(1440)$ is a pure three-quark baryon, $J / \Psi \rightarrow \bar{p} N^{*}(1440)$ should have the same Feynman diagram Fig.1 as for $J / \Psi \rightarrow \bar{p} p$. The only difference for the two processes is their quark wave functions and masses. Here we perform a calculation of the ratio between production rates of two processes by assuming simple three-quark wave functions for them. By comparing with experimental data, we can see whether the $N^{*}(1440)$ is produced more than the quark model prediction. 


\section{Formalism}

For the basic amplitude corresponding to Fig.1, we have

$$
\begin{aligned}
& \left\langle q_{i}, s_{i}, q_{i}^{\prime}, s_{i}^{\prime}, i=1,2,3|\hat{T}| J / \Psi^{(\Lambda)}\right\rangle \\
= & C_{0} \delta^{4}\left(P_{\Psi}-\sum_{i=1}^{3} q_{i}-\sum_{i=1}^{3} q_{i}^{\prime}\right) \cdot \epsilon_{\Psi}^{(\Lambda) \lambda} \cdot \frac{g_{\mu \lambda} g_{\nu \rho}+g_{\nu \lambda} g_{\mu \rho}+g_{\rho \lambda} g_{\mu \nu}}{\left(q_{1}+q_{1}^{\prime}\right)^{2}\left(q_{2}+q_{2}^{\prime}\right)^{2}\left(q_{3}+q_{3}^{\prime}\right)^{2}} \\
& \cdot \bar{u}\left(q_{1}^{\prime}, s_{1}^{\prime}\right) \gamma^{\mu} v\left(q_{1}, s_{1}\right) \bar{u}\left(q_{2}^{\prime}, s_{2}^{\prime}\right) \gamma^{\nu} v\left(q_{2}, s_{2}\right) \bar{u}\left(q_{3}^{\prime}, s_{3}^{\prime}\right) \gamma^{\rho} v\left(q_{3}, s_{3}\right)
\end{aligned}
$$

where $\epsilon_{\Psi}^{(\Lambda)}$ is the polarization four-vector of $J / \Psi$ with the helicity value $\Lambda, P_{\Psi}$ is the fourvector momentum of $J / \Psi, q_{i}^{\prime}, s_{i}^{\prime}\left(q_{i}, s_{i}\right)$ are the four-vector momenta and spin z-projection of quarks (anti-quarks), respectively. We have put all color matrix elements, QCD strong coupling constants, $J / \Psi$ decay constants, etc., into a single overall constant $C_{0}$.

The relation between the $J / \Psi \rightarrow \bar{p} N^{\prime}$ amplitude and the basic quark diagram amplitude Eq.(1) is

$$
\begin{aligned}
\mathcal{M}_{s_{z}, s_{z}^{\prime}}^{(\Lambda)} \equiv & \left\langle\Psi_{\bar{p}}\left(q, s_{z}\right) \Psi_{N^{\prime}}\left(q^{\prime}, s_{z}^{\prime}\right)|\hat{T}| J / \Psi^{(\Lambda)}\right\rangle \\
= & \sum_{s_{i}, s_{i}^{\prime}} \int \prod_{i=1}^{3} \frac{d \vec{q}_{i}}{(2 \pi)^{3} 2 q_{i}^{0}} \frac{d \vec{q}_{i}^{\prime}}{(2 \pi)^{3} 2 q_{i}^{\prime 0}}\left\langle\Psi_{\bar{p}}\left(q, s_{z}\right) \Psi_{N^{\prime}}\left(q^{\prime}, s_{z}^{\prime}\right) \mid q_{i}, s_{i}, q_{i}^{\prime}, s_{i}^{\prime}, i=1,2,3\right\rangle \\
& \cdot\left\langle q_{i}, s_{i}, q_{i}^{\prime}, s_{i}^{\prime}, i=1,2,3|\hat{T}| J / \Psi^{(\Lambda)}\right\rangle .
\end{aligned}
$$

Here $\left\langle\Psi_{\bar{p}}\left(q, s_{z}\right) \Psi_{N^{\prime}}\left(q^{\prime}, s_{z}^{\prime}\right) \mid q_{i}, s_{i}, q_{i}^{\prime}, s_{i}^{\prime}, i=1,2,3\right\rangle$ is the product of quark model wave functions of $\bar{p}$ and $N^{\prime}$ in momentum space, with constraints $\delta^{4}\left(q-q_{1}-q_{2}-q_{3}\right) \cdot \delta^{4}\left(q^{\prime}-q_{1}^{\prime}-q_{2}^{\prime}-q_{3}^{\prime}\right)$. The only difference between quark wave functions of the proton and $N^{*}(1440)$ is their spatial parts, which we assume to be simple harmonic-oscillator eigenfunctions in their center-of-mass (CM) systems, i.e.,

$$
\begin{aligned}
\Phi_{\bar{p}}\left(\vec{k}_{\rho}, \vec{k}_{\lambda}\right) & =\left(\frac{1}{\pi \alpha}\right)^{3 / 2} e^{-\frac{1}{2 \alpha}\left(\vec{k}_{\rho}^{2}+\vec{k}_{\lambda}^{2}\right)} \quad \text { for proton; } \\
\Phi_{N^{*}}\left(\vec{k}_{\rho}, \vec{k}_{\lambda}\right) & =\sqrt{3}\left(\frac{1}{\pi \alpha}\right)^{3 / 2}\left[1-\frac{1}{3 \alpha}\left(\vec{k}_{\rho}^{2}+\vec{k}_{\lambda}^{2}\right)\right] e^{-\frac{1}{2 \alpha}\left(\vec{k}_{\rho}^{2}+\vec{k}_{\lambda}^{2}\right)} \quad \text { for } \quad N^{*}(1440)
\end{aligned}
$$

where $\alpha=m \omega$ is the harmonic-oscillator parameter,

$$
\begin{aligned}
\vec{k}_{\rho} & =\frac{1}{\sqrt{6}}\left(\vec{k}_{1}+\vec{k}_{2}-2 \vec{k}_{3}\right), \\
\vec{k}_{\lambda} & =\frac{1}{\sqrt{2}}\left(\vec{k}_{1}-\vec{k}_{2}\right)
\end{aligned}
$$

with $\vec{k}_{1}, \vec{k}_{2}$, and $\vec{k}_{3}$ the three quark momenta in the CM system of their corresponding baryon, which are related to $\vec{q}_{i}$ or $\vec{q}_{i}^{\prime}$ by a Lorentz transformation.

In the $J / \Psi$ at rest system, the two baryon clusters are moving in opposite directions with highly relativistic speeds, each becoming very flat. Their spatial quark wave functions in this system are related to their CM wave functions as follows 10: 


$$
\Psi\left(\vec{q}_{\rho}, \vec{q}_{\lambda}\right)=\left|\frac{\partial\left(\vec{k}_{\rho}, \vec{k}_{\lambda}\right)}{\partial\left(\vec{q}_{\rho}, \vec{q}_{\lambda}\right)}\right|^{1 / 2} \Phi\left(\vec{k}_{\rho}, \vec{k}_{\lambda}\right)
$$

where

$$
\begin{aligned}
& \vec{q}_{\rho}=\frac{1}{\sqrt{6}}\left(\vec{q}_{1}+\vec{q}_{2}-2 \vec{q}_{3}\right), \\
& \vec{q}_{\lambda}=\frac{1}{\sqrt{2}}\left(\vec{q}_{1}-\vec{q}_{2}\right) .
\end{aligned}
$$

The spin and flavor wavefunctions of the proton and $N^{*}(1440)$ are the same, i.e.,

$$
\Psi_{S F}^{1}=\Psi_{S F}^{2}=\frac{1}{\sqrt{2}}\left(\chi^{\rho} \phi^{\rho}+\chi^{\lambda} \phi^{\lambda}\right)
$$

where $\chi^{\rho}$ and $\chi^{\lambda}$ are the mixed-symmetry pair spin- $\frac{1}{2}$ wavefunctions. For example, we have

$$
\begin{aligned}
\chi_{\frac{1}{2}, \frac{1}{2}}^{\rho} & =-\frac{1}{\sqrt{6}}\{|\uparrow \downarrow \uparrow>+| \downarrow \uparrow \uparrow>-2 \mid \uparrow \uparrow \downarrow>\}, \\
\chi_{\frac{1}{2}, \frac{1}{2}}^{\lambda} & =\frac{1}{\sqrt{2}}\{|\uparrow \downarrow \uparrow>-| \downarrow \uparrow \uparrow>\}
\end{aligned}
$$

for the case of the total spin $\frac{1}{2}$ and its projection $\frac{1}{2}$. The flavor wavefunctions $\phi^{\rho}$ and $\phi^{\lambda}$ are exactly analogous to that of the spin wavefunctions but in isospin space of $u$ - $d$ quarks.

We perform the calculation in the $J / \Psi$ rest system. For $J / \Psi$ produced in $e^{+} e^{-}$annihilation, its helicity is limited to be $\Lambda= \pm 1$. The components in Eq.(1) can be expressed more explicitly as

$$
\begin{aligned}
& \epsilon_{\Psi}^{( \pm)}=\left(0 ; \mp \frac{1}{\sqrt{2}},-\frac{i}{\sqrt{2}}, 0\right) \\
& \bar{u}\left(q_{i}^{\prime}, s_{i}^{\prime}\right) \gamma^{0} v\left(q_{i}, s_{i}\right)=0 \\
& \bar{u}\left(q_{i}^{\prime}, s_{i}^{\prime}\right) \vec{\gamma} v\left(q_{i}, s_{i}\right)=\frac{E_{q}+m_{q}}{2 m_{q}}\left\langle s_{i}^{\prime}\left|\left(1+\frac{\left|\vec{q}_{i}\right|^{2}}{\left(E_{q}+m_{q}\right)^{2}}\right) \vec{\sigma}-\frac{2\left(\vec{\sigma} \cdot \vec{q}_{i}\right) \vec{q}_{i}}{\left(E_{q}+m_{q}\right)^{2}}\right| s_{i}\right\rangle
\end{aligned}
$$

where $E_{q}$ and $m_{q}$ are the energy and mass of the quark.

In Eq.(2), the integration over $\prod_{i=1}^{3} d \vec{q}_{i} d \vec{q}_{i}^{\prime}$ with two $\delta^{4}$ functions can be reduced to a ten-dimension integration which we carry out numerically with the adaptive multidimensional Monte-Carlo integration program RIWIAD of CERN Program Library. From these amplitudes $\mathcal{M}_{s_{z}, s_{z}^{\prime}}^{(\Lambda)}$, we can get the decay cross section for $J / \Psi^{(\Lambda)} \rightarrow \bar{p} N^{\prime}$ as

$$
d \Gamma\left(J / \Psi^{(\Lambda)} \rightarrow \bar{p} N^{\prime}\right)=\frac{1}{32 \pi^{2}}\left\{\left|\mathcal{M}_{\frac{1}{2}, \frac{1}{2}}^{(\Lambda)}\right|^{2}+\left|\mathcal{M}_{\frac{1}{2},-\frac{1}{2}}^{(\Lambda)}\right|^{2}+\left|\mathcal{M}_{-\frac{1}{2}, \frac{1}{2}}^{(\Lambda)}\right|^{2}+\left|\mathcal{M}_{-\frac{1}{2},-\frac{1}{2}}^{(\Lambda)}\right|^{2}\right\} \frac{|\vec{q}|}{M_{\Psi}^{2}} d \Omega
$$

with $\Omega$ as the solid angle of $\vec{q}$.

The calculation of $J / \Psi \rightarrow \bar{N}^{*} N^{*}$ is similar, just replacing quark radial wavefunction of the anti-proton by that of the $\bar{N}^{*}(1440)$. With formulas above, the calculation of the decay cross sections is straightforward though tedious. 


\section{Numerical results and discussion}

In our quark model calculation, there are three parameters, i.e., the constituent quark mass $m_{q}$, the harmonic-oscillator parameter $\alpha$ and an overall normalization factor $C_{0}$. The relation between $\alpha$ and the nucleon radius $r_{0}$ is $\alpha=1 / r_{0}^{2}$. In most quark model calculations [3, 10, 11, 12, 13, the quark mass $m_{q}$ has been chosen in the range of $220 \sim 340$ $\mathrm{MeV}$, and $\alpha$ in the range of $0.06 \sim 0.22 \mathrm{GeV}^{2}$ which corresponds to the nucleon radius in the range of $0.42 \sim 0.8 \mathrm{fm}$. In the following, we limit our parameters in these ranges.

The $J / \Psi$ decay cross sections for $\bar{p} p, \bar{p} N^{*}$ and $\bar{N}^{*} N^{*+}$ can be expressed as

$$
\begin{aligned}
\frac{d \Gamma\left(J / \Psi^{( \pm)} \rightarrow \bar{p} p\right)}{d \Omega} & =N_{\bar{p} p}\left(1+\alpha_{p} \cos ^{2} \theta\right), \\
\frac{d \Gamma\left(J / \Psi^{( \pm)} \rightarrow \bar{p} N^{*}\right)}{d \Omega} & =R_{*} N_{\bar{p} p}\left(1+\alpha_{*} \cos ^{2} \theta\right), \\
\frac{d \Gamma\left(J / \Psi^{( \pm)} \rightarrow \bar{N}^{*} N^{*+}\right)}{d \Omega} & =R_{* *} N_{\bar{p} p}\left(1+\alpha_{* *} \cos ^{2} \theta\right) .
\end{aligned}
$$

Here $N_{\bar{p} p}$ is a constant direct related to the experimental branching ratio of $J / \Psi \rightarrow \bar{p} p$ and can be used to fix the overall normalization constant $C_{0}$. The experimental value for $\alpha_{p}$ is $(0.62 \pm 0.11) 14$ and can be used to put further limit on the range of parameters $\alpha$ and $m_{q}$. The shaded area in Fig.2 shows the range allowed by one standard deviation of the experimental $\alpha_{p}$ value.

In order to investigate the importance of the Lorentz contraction effect, we have also performed the calculation by ignoring this effect, i.e., assuming $\vec{k}_{\rho}=\vec{q}_{\rho}$ and $\vec{k}_{\lambda}=\vec{q}_{\lambda}$. The resulted $\left(\alpha, m_{q}\right)$ area allowed by one standard deviation of the experimental $\alpha_{p}$ value is shown in Fig. 2 by the area surrounded by the solid line. One can see that the Lorentz contraction effect is very large and cannot be ignored.

With $\left(\alpha, m_{q}\right)$ values in the shaded area of Fig.2, our quark model calculation predicts $\alpha_{*}=0.36 \pm 0.08, \alpha_{* *}=0.08 \pm 0.05, R_{*}=2.1 \sim 4.8$ and $R_{* *}=2.0 \sim 24.0$. Mixings between the ground state and the radially excited states 12 will not change our result much due to the relative negative sign of mixings for the proton and $N^{*}(1440)$.

There are no experimental data on $\bar{p} N^{*}$ and $\bar{N}^{*} N^{*}$ channels yet. However from both BESI[7] and MARKII[15] experiments, there is a clear peak around $1.5 \mathrm{GeV}$ in the $\pi N$ invariant mass in $J / \psi \rightarrow \bar{p} N \pi$ processes, although no partial wave analyses were performed. Very recently BESII has finished data-taking for 50 million more $J / \psi$ events, which is about two order of manitude more statistics than MARKII data and one order of magnitude more statistics than BESI data. With such statistics, partial wave analyses of relevant channels are possible. New experimental results on $J / \Psi \rightarrow \bar{p} p, J / \Psi \rightarrow \bar{p} N^{*}$ and $J / \Psi \rightarrow \bar{N}^{*} N^{*}$ will help us to narrow down the quark model $\left(\alpha, m_{q}\right)$ parameters and study the nature of $N^{*}$. If the $J / \Psi \rightarrow \bar{p} N^{*}$ production rate is significantly larger than our quark model prediction, it may indicate that the $N^{*}$ is a hybrid $[9]$; if $J / \Psi \rightarrow \bar{p} N^{*}$ production rate is significantly smaller than our prediction, then it may indicate that the $N^{*}$ contains a large component of $\pi N$ in its internal structure [13]. For a more quantitative statement, concrete theoretical calculations for hybrid and molecule baryon production are needed. 


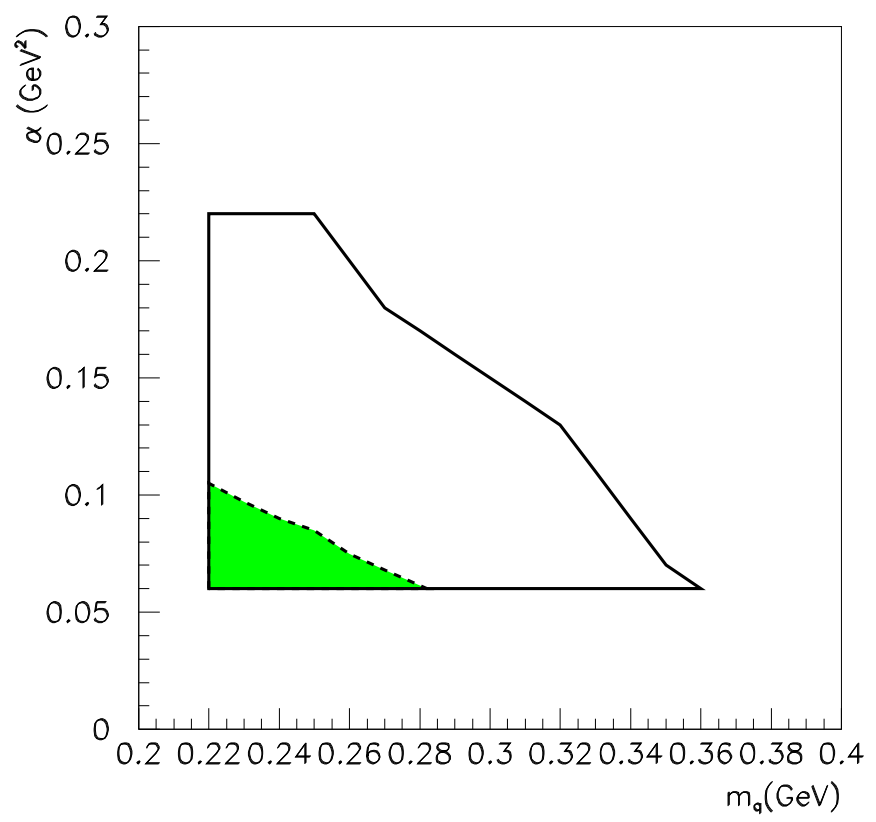

Figure 2: The constrained area for parameters $\left(\alpha, m_{q}\right)$ from experimental data $\alpha_{p}=$ $0.62 \pm 0.11[14]$. The shaded area is the result with the Lorentz contraction effect; the area surrounded by the solid line is the result ignoring the Lorentz contraction effect. 
Acknowledgement: We thank D.V.Bugg, Y.B.Dong, L.Kisslinger, H.B.Li, Y.W.Yu and Z.Y.Zhang for useful discussions and comments. This work is partly supported by

National Science Foundation of China under contract Nos. 19991487 and 19905011.

\section{References}

[1] V.Burkert, Proceedings, Perspectives in the structure of hadronic systems, ed by M.N.Harakeh et al., Plenum Press New York 1994.

[2] Particle Data Group, Euro. Phys. J. C15 (2000) 1.

[3] N.Isgur and G.Karl, Phys. Rev. D18 (1978) 4187; ibid. 19 (1979) 2653;

S.Capstick and N.Isgur, Phys. Rev. D 34 (1986) 2809;

L.Glozman et al., Phys. Rev. D58 (1998) 094030;

R.Bijker, F.Iachello and A.Leviatan, Ann. Phys. (N.Y.) 236 (1994) 69;

K.F.Liu and C.W.Wong, Phys. Rev. D28 (1983) 170.

[4] T.Barnes and F.E.Close, Phys. Lett. B123 (1983) 89;

E.Golowich, E.Haqq and G.Karl, Phys. Rev. D28 (1983) 160;

L.Kisslinger and Z.P.Li, Phys. Rev. D 51 (1995) R5986.

[5] R.Workman, Few Body Syst. Suppl. 11 (1999) 94.

[6] B.S.Zou, Nucl. Phys. A675 (2000) 167; Nucl. Phys. A684 (2001) 330.

[7] BES Collaboration, H.B.Li et al., Nucl. Phys. A675 (2000) 189c; B.S.Zou et al., hep-ph/0004220.

[8] C.Carimalo, Int. J. Mod. Phys. A2 (1987) 249.

[9] S.Capstick and P.Page, Phys. Rev. D 60 (1999) 111501.

[10] S.Capstick and B.D.Keister, Phys. Rev. D51 (1995) 3598.

[11] F.Foster and G.Hughs, Z. Phys. C14 (1982) 123;

C.Hayne and N.Isgur, Phys. Rev. D25 (1982) 1944;

Z.Y.Zhang et al., Nucl. Phys. A625 (1997) 59.

[12] S.Capstick, Phys. Rev. D46 (1992) 1965.

[13] Y.B.Dong, et. al., Phys. Rev. C60 (1999) 035203.

[14] DM2 Collaboration, Nucl. Phys. B292 (1987) 653.

[15] M.W.Eaton, et al., Phys. Rev. D29 (1984) 804. 